\title{
Terapias farmacológicas para la COVID-19
}

\section{Pharmacological treatments for COVID-19}

José Antonio Morales Fernández, * Alejandra Xóchitl Cruz Salgado,* José Enrique Chávez Aguilar,* Edson Erivan Mosqueda Martínez, * Deyanira Gutiérrez Bautista, * Hiram Joaquín Vilchis, * Ikky Omar Ramírez Velázquez, ${ }^{\ddagger}$ Luis Ángel Perón Medina, ${ }^{\ddagger}$ Zurisadai Raquel García Osorno, ${ }^{\ddagger}$ Leonardo Martín Vásquez Martínez, * Yazmín Valadez González, ${ }^{*}$ Luis Alberto Cortázar Maldonado, ${ }^{\ddagger}$ Xcarelt Vite Velázquez, ${ }^{\ddagger}$ Antonio Humberto Ángel Ambrocio, * Miguel Leonardo García León,* Patricia Bautista Carbajal, ${ }^{*}$ Jorge Baruch Díaz Ramírez, ${ }^{\ddagger}$ Rosa María Wong Chew*

Citar como: Morales FJA, Cruz SAX, Chávez AJE, Mosqueda MEE, Gutiérrez BD, Joaquín VH et al. Terapias farmacológicas para la COVID-19. Acta Med Grupo Angeles. 2021; 19 (3): 408-420. https://dx.doi.org/10.35366/101740

\section{Resumen}

El virus SARS-CoV-2 es el coronavirus que causa la enfermedad por coronavirus 19 (COVID-19), ésta es una enfermedad de preocupación mundial y declarada pandemia en el 2020. Para el adecuado control se han tomado medidas de protección personal e investigado una gran variedad de medicamentos que actúen en las distintas etapas del proceso patogénico para evitar la adhesión, replicación y progresión del virus en los diferentes estadios de la enfermedad, así como la generación de anticuerpos neutralizantes efectivos contra la misma. El surgimiento de las variantes genéticas ha generado la necesidad del avance en las tecnologías para la elaboración e investigación de nuevos blancos terapéuticos. Actualmente, no se cuenta con ningún medicamento específico para el tratamiento farmacológico de la COVID-19; sin embargo, se utilizan diferentes medicamentos que han evitado la progresión de la enfermedad, la recuperación, así como la disminución de la mortalidad y aumento de la supervivencia.

Palabras clave: SARS-CoV-2, COVID-19, coronavirus, tratamiento, blancos farmacológicos.
* Laboratorio de Investigación en Enfermedades Infecciosas.

${ }^{\ddagger}$ Clínica de Atención Preventiva del Viajero.

División de Investigación, Facultad de Medicina, UNAM.

Correspondencia:

Rosa María Wong Chew

Correo electrónico: rmwong@unam.mx

Aceptado: 23-06-2021.

www.medigraphic.com/actamedica
Abstract

SARS-CoV-2 is the coronavirus that causes coronavirus disease 2019 (COVID-19), which is a disease of global concern and declared as a pandemic in 2020. For adequate control, personal protection measures have been taken and a great variety of drugs have been investigated to act in the pathogenic process to prevent the adhesion, replication and progression of the virus in the different stages of the disease, as well as the generation of effective neutralizing antibodies against it. The emergence of genetic variants has generated the need for advances in technologies for the development and research of new therapeutic targets. Currently, there is no specific drug for the pharmacological treatment of COVID-19, however, different drugs are used that have prevented the progression of the disease, recovery of illness, decreased mortality and increased survival.

Keywords: SARS-CoV-2, COVID-19, coronavirus, treatment, pharmacological targets.

\section{INTRODUCCIÓN}

El SARS-CoV-2 es un betacoronavirus envuelto, perteneciente al subgénero Sarbecovirus, subfamilia Orthocoronavirinae, y agente causal de la enfermedad por coronavirus 2019 (COVID-19, por sus siglas en inglés). Esta enfermedad surgió en diciembre del 2019 en Wuhan, China como una neumonía severa que se propagó globalmente, lo que originó la pandemia por COVID-19, declarada el 11 de marzo del 2020. Este virus posee un genoma de ARN monocatenario no segmentado de sentido positivo que codifica para 16 proteínas no estructurales y cuatro estructurales: la glicoproteína espiga (S), responsable de la unión 
y fusión del virus con las membranas celulares; la proteína de membrana $(M)$, responsable de la liberación de la partícula viral y la formación de su envoltura; la proteína de nucleocápside (N) y la proteína de envoltura (E) ${ }^{1}$ (Figura 1).

La pandemia por SARS-CoV-2 ha causado un gran número de decesos alrededor del mundo, se han estudiado y empleado una gran variedad de fármacos para intentar controlar la infección, pero actualmente no existe un tratamiento específico. Sin embargo, algunos de los fármacos empleados hasta ahora, han contribuido al mejor manejo de las complicaciones graves asociadas a la enfermedad, así como a disminuir la mortalidad. El objetivo de este artículo es conocer los principales fármacos que se han empleado a lo largo de la pandemia por la COVID-19, haciendo especial hincapié en los blancos terapéuticos durante el proceso patogénico viral, sus indicaciones dependiendo de la gravedad, así como aquellos que han sido aprobados o rechazados para su empleo terapéutico.

\section{BLANCOS FARMACOLÓGICOS PARA LA INFECCIÓN POR EL VIRUS SARS-COV-2}

El SARS-CoV-2 ingresa al hospedero a través del receptor de la enzima convertidora de angiotensina 2 (ECA2), expresado diferencialmente en distintos tejidos corporales como son: el epitelio de la mucosa nasal, oral, nasofaringe, pulmonar, gastrointestinal, tegumentario, renal, vascular, hepático, cerebral, testicular, en linfonodos y en el bazo. ${ }^{2}$ La unión del virus al ECA2 es el primer paso de la infección viral. Para ello, la proteína $S$ utiliza sus dos subunidades funcionales: la subunidad S1 que permite la unión al receptor celular (ECA2) y la subunidad S2, que facilita la fusión del virus con las membranas de la célula hospedera. Una vez unido al receptor ECA2, el complejo formado se procesa proteolíticamente mediante la proteasa de transmembrana tipo 2 (TMPRSS2) celular, lo que facilita la escisión de ECA2 y genera la activación de $S$, iniciando de esta forma el proceso de unión-fusión del virus con la membrana celular. ${ }^{3}$ En este punto de la infección se ha sugerido que los medicamentos como la cloroquina e hidroxicloroquina podrían inhibir la fusión y glicosilación de la proteína $\mathrm{S}$ del virión con el receptor ECA2, así como la ivermectina, que podría tener un papel en la inhibición de esta adhesión y de la inhibición de las proteínas de transporte nuclear del hospedero, importina alfa/beta-1.4,5

Una vez completada la etapa de unión/fusión, el material genético viral es liberado dentro del citoplasma donde se realiza el proceso de transcripción, la secuencia de un gen se copia para hacer una molécula de ARN; replicación, se duplica el nuevo material genético; y traducción, se generan las diferentes proteínas del virión a partir de un ARNm, para la formación de nuevos viriones. Durante estos procesos, se propusieron al análogo de adenosina, remdesivir, para inhibir la replicación del virión del SARSCoV-2 al incorporarse a la ARN polimerasa, y a los inhibidores de proteasas, lopinavir/ritonavir, cuya función es inhibir la sección proteica del material genético del virión para así evitar el ensamblaje y conformación de las distintas estructuras víricas. ${ }^{4,5}$

Cuando se logra la conformación de viriones por las células hospederas, éstos son liberados al torrente sanguíneo, donde son reconocidos por diferentes células inmunológicas, como las células dendríticas, linfocitos CD4+ Th1, macrófagos y neutrófilos para su eliminación; así como la estimulación de linfocitos B para la producción de anticuerpos dirigidos contra el virión. En caso de sobrepasar los mecanismos de defensa inicial, el organismo hospedero inicia la producción descontrolada de citocinas proinflamatorias, entre las que destacan las interleucinas (IL) 1, 2, 6, interferón gamma (IFN- $\gamma$ ) y el factor de necrosis tumoral alfa (TNF- $\alpha$ ), que generan una respuesta citotóxica a través de patrones moleculares asociados a daño (DAMPs) y a patógenos (PAMPs), una tormenta de citocinas y la subsecuente falla de órganos múltiples. En estos diferentes mecanismos destacan la colchicina, cuyo mecanismo propuesto fue la inhibición de la quimiotaxis, inhibir la formación del inflamasoma y de la IL-1; los inhibidores de IL-1 (anakinra), inhibidores de IL-6 (tocilizumab); y los productos con anticuerpos contra el SARS-CoV-2 para crear una mayor contención del virión. ${ }^{4,5}$

Sin embargo, aunque muchos de estos blancos terapéuticos fueron prometedores al inicio de la pandemia, actualmente se ha descartado el uso de algunos y se ha recomendado el uso de otros por la Organización Mundial de la Salud (OMS) y las distintas agencias regulatorias de cada país para disminuir la mortalidad y mejorar la super-

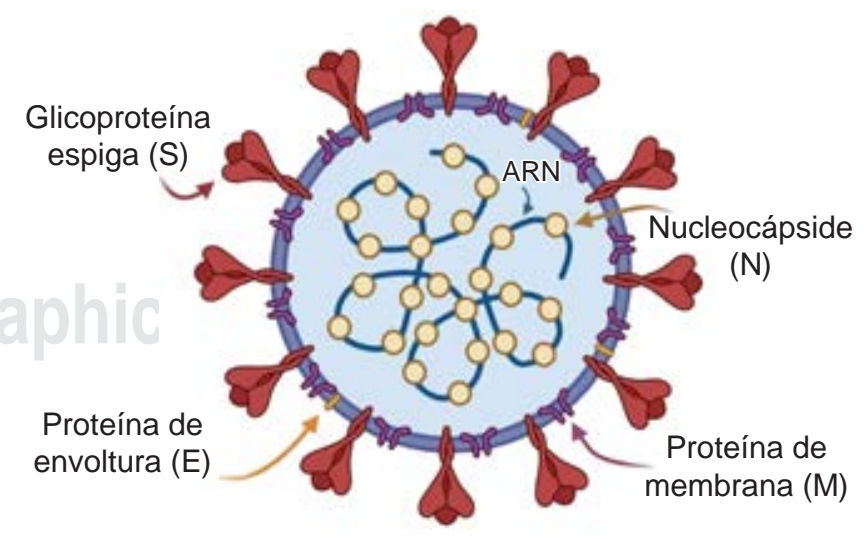

Figura 1: Estructura del virión del SARS-CoV-2 en la que se distinguen las principales proteínas que la conforman. 
vivencia de los pacientes que desarrollan la enfermedad por el virus SARS-CoV-2.

\section{TRATAMIENTO HOSPITALARIO DE COVID-19 GRAVE/SEVERO}

\section{COVID-19 grave o severo}

El espectro clínico de la infección por SARS-CoV-2 varía de un curso asintomático hasta una enfermedad crítica. La sintomatología incluye fiebre, tos, dolor de garganta, malestar general o mialgias; algunos pacientes también debutan con síntomas gastrointestinales como anorexia, diarrea y náuseas. ${ }^{6}$ Se ha reportado que $68 \%$ de los pacientes presenta anosmia y disgeusia. ${ }^{6}$ La enfermedad severa usualmente comienza una semana después del inicio de la sintomatología; la disnea es el hallazgo más frecuente en la enfermedad severa y por lo regular está acompañada de hipoxemia. A su vez, la mayor parte de los pacientes con enfermedad severa por COVID-19 desarrollan insuficiencia respiratoria progresiva. Estos pacientes frecuentemente cumplen con criterios para síndrome de distrés respiratorio agudo, definido como la aparición de infiltrados bilaterales, hipoxemia severa y edema pulmonar que no se explica por falla cardiaca o por sobrecarga hídrica. Es común el desarrollo de complicaciones tromboembólicas, sepsis, falla cardiaca y exacerbación de comorbilidades preexistentes, así como desórdenes en el sistema nervioso central y periférico. ${ }^{6-9}$

\section{Oxigenación y ventilación}

La saturación de oxígeno $\left(\mathrm{SatO}_{2}\right)$ óptima en personas con COVID-19 es incierta. No obstante, la meta de $\mathrm{SatO}_{2}$ debe ser de 92 a 96\%, considerando la evidencia indirecta de la experiencia en pacientes sin COVID-19, en donde saturaciones menores a $92 \%$ o mayores a $96 \%$ pueden ser dañinas. ${ }^{9}$

Los sistemas de ventilación de bajo flujo proporcionan menos de $40 \mathrm{~L} /$ min de oxígeno, por lo que no proporciona la totalidad del gas inspirado y parte del volumen inspirado es tomado del medioambiente. Todos estos dispositivos utilizan un borboteador que funciona como reservorio de agua para humidificar el oxígeno inspirado. Los dispositivos de bajo flujo más frecuentemente utilizados son las puntas nasales, máscara simple de oxígeno y máscara simple con reservorio. ${ }^{10}$ El uso de sistemas de ventilación de bajo flujo se debe utilizar en aquellos pacientes con hipoxemia sin signos respiratorios de urgencia (frecuencia respiratoria mayor a 24 respiraciones por minuto, saturación de oxígeno menor a $90 \%$, disnea y fiebre). ${ }^{11}$

Por otra parte, la oxigenoterapia de alto flujo permite administrar un flujo de gas totalmente acondicionado hasta a $60 \mathrm{~L} /$ min mediante cánulas nasales, obteniendo una rápida mejoría de los síntomas debido a diferentes mecanismos como, por ejemplo, una reducción de la resistencia de la vía aérea superior, cambios en el volumen circulante y la generación de cierto grado de presión positiva. ${ }^{12}$ Se recomienda utilizar sistemas de ventilación de alto flujo en pacientes con hipoxemia y datos de urgencia como en el caso de insuficiencia respiratoria aguda (saturación menor a 90\%, taquipnea y disnea, a pesar del uso de dispositivos de bajo flujo). Se han observado mayores beneficios en días libres de ventilación con el uso de cánula nasal de alto flujo, en comparación con los dispositivos de presión positiva en el manejo de la insuficiencia respiratoria aguda hipoxémica; siendo de 24 días libres de ventilador para el uso de la cánula nasal y de 19 días libres de ventilador para la ventilación con presión positiva; así como una disminución de la mortalidad a 90 días con el uso de la cánula nasal. ${ }^{13,14}$

La ventilación mecánica es el proceso, mediante diferentes dispositivos, en donde se sustituye, total o parcialmente y de forma temporal, la ventilación espontánea; es decir, el intercambio de oxígeno y $\mathrm{CO}_{2}$ entre el aire ambiente y el alvéolo. Dicho procedimiento se realiza tanto de forma invasiva, a través de intubación endotraqueal y aplicación de presión positiva con un ventilador mecánico. ${ }^{15}$

Es esencial monitorizar de cerca a aquellos pacientes con hipoxemia en busca de signos de descompensación respiratoria para evaluar el uso de ventilación mecánica en quienes cumplan criterios para el manejo avanzado de la vía aérea, como son la taquipnea persistente mayor a 30 rpm, saturación menor a $90 \%$ pese a oxígeno suplementario $\left(\mathrm{FiO}_{2}\right)$, insuficiencia respiratoria aguda y datos de choque. ${ }^{16}$

Así mismo, se ha observado que la pronación de pacientes con ventilación mecánica ha mejorado su oxigenación, aumentando el índice $\mathrm{PaO}_{2} / \mathrm{FiO}_{2}$. Se recomienda utilizar presiones finales de espiración positiva altas (PEEP, por sus siglas en inglés) para prevenir el colapso alveolar, mejorar la oxigenación y minimizar las atelectasias como resultado de lesión pulmonar inducida por ventilador. ${ }^{17,18}$ La pronación de los pacientes está contraindicado cuando se requiere intubación inmediata, pacientes hemodinámicamente inestables y en pacientes con cirugía de abdomen reciente.

\section{Manejo hemodinámico}

Se debe comenzar con fluidoterapia de manera conservadora en aquellos pacientes sin datos de hipoperfusión. En pacientes que requieran reanimación hídrica o manejo hemodinámico del choque se recomienda ser tratados como en el choque séptico. ${ }^{19}$

El uso de soluciones cristaloides balanceadas debe llevarse a cabo en primera instancia y se debe evitar el uso de coloides. ${ }^{20,21}$ 
En caso de llegar a requerir el uso de vasopresores, se recomienda iniciar con norepinefrina, monitorizando en todo momento la presión arterial media (PAM). Si se logra mantener una PAM por debajo de $65 \mathrm{mmHg}$ se debe agregar vasopresina (por arriba de 0.03 unidades/minuto) o epinefrina. Se espera la aparición de falla cardiaca y persistencia de datos de hipoperfusión después de una adecuada terapia con soluciones cristaloides y aminas vasoactivas, por lo que se recomienda la utilización de dobutamina en estos casos.

\section{Terapia antitrombótica en pacientes hospitalizados}

La infección por SARS-CoV-2 se ha asociado con estados protrombóticos, con elevación de fibrina y sus productos de degradación como fibrinógeno y dímero D. En algunos estudios, la elevación de estos marcadores se asocia a peores resultados clínicos. ${ }^{22}$

Se recomienda el uso de heparina de bajo peso molecular y heparina no fraccionada sobre los anticoagulantes orales, debido a que los primeros tienen una vida media menor, pueden ser administradas por vía intravenosa o subcutánea y tienen menos interacciones farmacológicas. ${ }^{23}$ Se debe evitar el uso de anticoagulantes en pacientes con COVID-19 leve y con manejo ambulatorio, a menos que haya razón o indicación necesaria para su uso.

\section{Manejo terapéutico de pacientes con COVID-19 grave/severo}

En los pacientes con hipoxemia y sin requerimientos de oxígeno, se recomienda el uso de remdesivir y evitar el uso de dexametasona y tocilizumab. ${ }^{24}$

En los pacientes que requieren oxígeno suplementario a bajo flujo, se debe iniciar manejo con dexametasona y remdesivir. Por otra parte, en aquellos pacientes con requerimientos bajos de oxígeno, pero con marcadores inflamatorios altos, se recomienda un incremento rápido en los requerimientos de oxígeno e iniciar tratamiento con tocilizumab. ${ }^{25,26}$

En pacientes con requerimientos de oxígeno con dispositivos de alto flujo, se recomienda utilizar dexametasona. Si el paciente se encuentra dentro de las primeras 24-48 horas de su ingreso a la $\mathrm{UCl}$, se recomienda iniciar con tocilizumab. También se puede considerar el uso de remdesivir en estos pacientes, pero se deben priorizar aquellos que requieran oxígeno a bajo flujo. ${ }^{27,28}$

Pacientes con ventilación mecánica: se recomienda el uso de dexametasona. Si el paciente se encuentra dentro de sus primeras 24 horas de su ingreso a la $\mathrm{UCl}$, se recomienda iniciar con tocilizumab. ${ }^{26,29}$

En la Figura 2 se propone un algoritmo sobre el manejo terapéutico de los pacientes con COVID-19.

\section{ANTIRRETROVIRALES, ANTIBIÓTICOS Y ANTIPARASITARIOS}

Una gran variedad de medicamentos, empleados en diversas enfermedades infecciosas preexistentes, han sido motivo de estudio como blancos terapéuticos para el manejo de la COVID-19. Entre los más significativos destacan:

\section{Remdesivir}

Es un profármaco análogo del nucleótido adenosina que se metaboliza en las células del hospedero para la formación del nucleósido trifosfato, el cual es farmacológicamente activo y, por lo tanto, inhibe la replicación del virión del SARS-CoV-2 al incorporarse a la ARN polimerasa ${ }^{30}$ (Figura 3).

Este profármaco ha sido aprobado, por distintos organismos como la Organización Mundial de la Salud (OMS) y la Food and Drug Administration (FDA), para el tratamiento de la COVID-19 en pacientes hospitalizados y evidencia de infección del tracto respiratorio inferior, tanto en pediátricos como adultos, a partir de los 12 años y por arriba de los $40 \mathrm{~kg}$ de peso corporal; no se han documentado en estudios clínicos su uso con parámetros menores; sin embargo, la FDA lo ha aprobado, de igual modo, en los pacientes pediátricos menores de 12 años y mayores de $3.5 \mathrm{~kg}$ de peso corporal. ${ }^{31}$

Su dosificación es vía intravenosa (IV) con 200 mg en el día uno y de 100 mg del día dos al 10, evitando sobrepasar los 10 días de tratamiento. ${ }^{32}$

Entre sus principales efectos adversos destacan las molestias gastrointestinales, elevación de transaminasas y del tiempo de protrombina, así como hipersensibilidad al medicamento. Su uso debe ser suspendido en caso de una elevación importante de ALT mayor a 10 veces su valor normal y/o con datos clínicos de inflamación hepática. ${ }^{32}$

El empleo combinado de remdesivir con $4 \mathrm{mg}$ de baricitinib (inhibidor selectivo y reversible de la Janus cinasa, JAK$1 / 2$ ) vía nasal o nasogástrica por 14 días, ha demostrado una mayor eficacia, que el uso único de remdesivir, en la mejoría clínica de pacientes con COVID-19 y en la reducción de la recuperación de la enfermedad en pacientes hospitalizados. ${ }^{33}$

No se recomienda la administración de remdesivir en pacientes con filtrado glomerular $(\mathrm{FG})<30 \mathrm{~mL} / \mathrm{min}$ por su mecanismo de excreción renal; así como en pacientes embarazadas, a menos que esté justificado su uso. ${ }^{34}$

\section{Lopinavir/ritonavir}

Son inhibidores de proteasas, enzimas encargadas de seccionar las proteínas sintetizadas a partir del material genético del virus para que se puedan ensamblar y conformar las distintas estructuras del virión; entre estas enzimas 


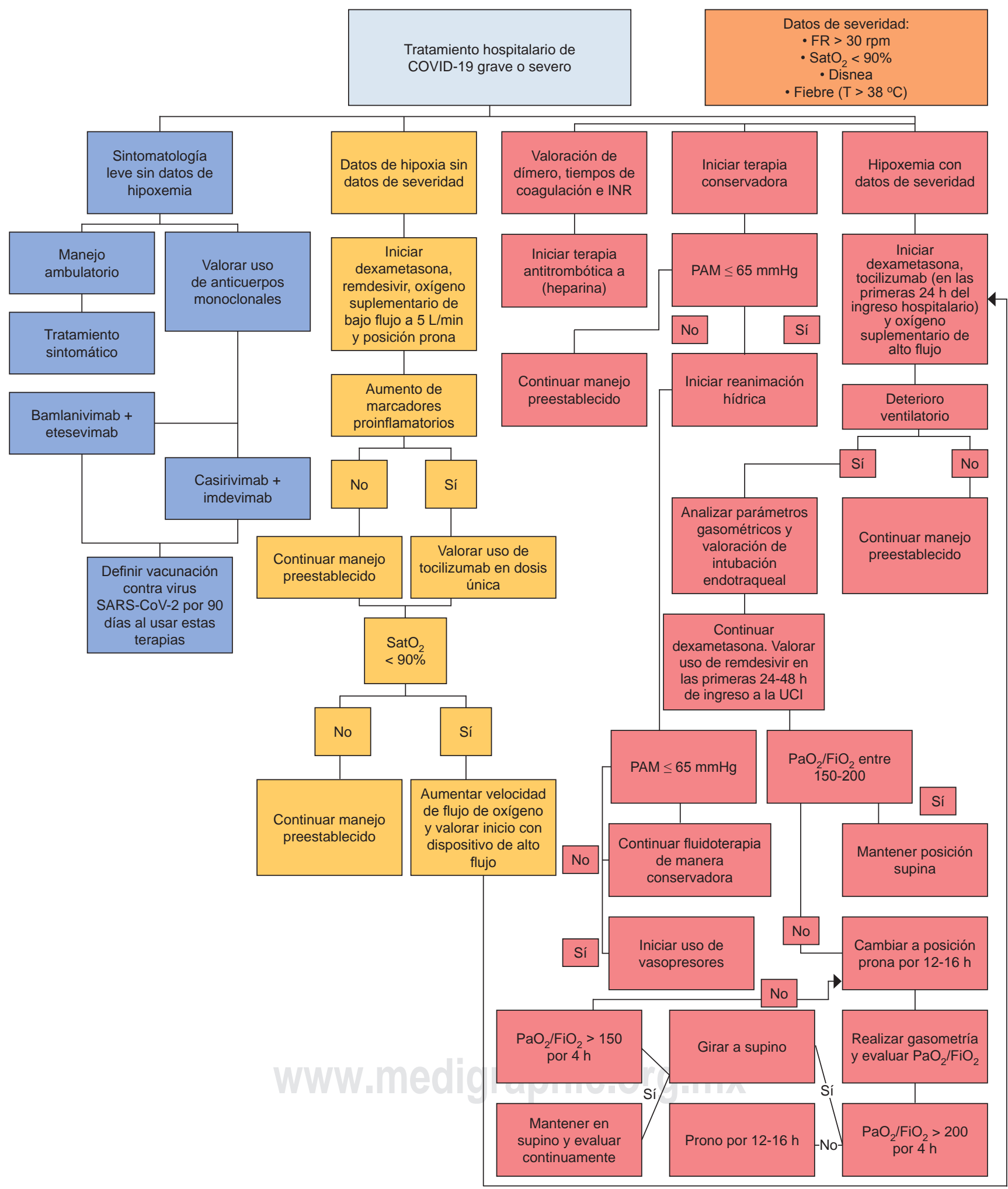

Figura 2: Algoritmo terapéutico propuesto para el manejo de los pacientes con COVID-19 de acuerdo con los fármacos y blancos terapéuticos aprobados por las agencias regulatorias y la OMS.

$\mathrm{FR}$ = frecuencia respiratoria; $\mathrm{SatO}_{2}=$ saturación de oxígeno; INR = International Normalized Ratio; $\mathrm{PAM}=$ presión arterial media; UCI = Unidad de Cuidados Intensivos; $\mathrm{PaO}_{2}=$ presión arterial de oxígeno; $\mathrm{FiO}_{2}=$ fracción inspiratoria de oxígeno. 
destacan la proteasa similar a la 3-quimotripsina (3CLpro) y la proteasa similar a la papaína (PLpro) ${ }^{34}$ (Figura 3).

Los estudios RECOVERY demostraron que el uso de inhibidores de proteasas como lopinavir/ritonavir en dosis de 400 y $100 \mathrm{mg}$, respectivamente, por vía oral durante 10 días o hasta la remisión, no mostraron reducciones en la mortalidad, de la estancia hospitalaria ni de la progresión de la enfermedad por COVID-19 en pacientes hospitalizados; por lo que la OMS descartó su empleo como blanco terapéutico de la enfermedad. ${ }^{35}$

Entre sus efectos adversos se encuentran principalmente alteraciones gastrointestinales (diarrea, náusea o vómito), prolongación del QT y hepatotoxicidad. ${ }^{34}$

\section{Cloroquina o hidroxicloroquina}

La cloroquina e hidroxicloroquina son fármacos análogos de la 4-aminoquinolona que han sido empleados en el tratamiento de otras enfermedades como la malaria/paludismo y distintas enfermedades autoinmunes. ${ }^{34}$

Su empleo al inicio de la pandemia para el tratamiento de la COVID-19 fue controvertido, debido a que su mecanismo de acción propuesto es la inhibición de la fusión y glicosilación de la proteína S del SARS-CoV-2 con el receptor de la enzima convertidora de angiotensina 2 (ACE2) de las células del hospedero ${ }^{34}$ (Figura 3).

En diferentes estudios clínicos de RECOVERY, se observó que el uso único de cloroquina e hidroxicloroquina a una dosis de $400 \mathrm{mg}$ dos veces al día durante siete días o el uso de 400 mg de hidroxicloroquina dos veces al día, más el uso de una dosis de 500 mg de azitromicina al día, no demostró efecto preventivo de pacientes sanos expuestos al virus, ni efecto terapéutico para la infección por SARS-CoV-2 en pacientes hospitalizados; por lo cual, la OMS descartó su uso como blanco terapéutico de la COVID-19. ${ }^{36,37}$

Los efectos adversos son mayores y no presentan beneficio alguno en el tratamiento, entre los que se incluyen la alteración cardiaca con prolongación del QT, torsades de pointes, arritmias ventriculares, hipoglucemia, rash, náusea, retinopatía y supresión de médula ósea. ${ }^{34}$

\section{Azitromicina}

Es un antibiótico de amplio espectro perteneciente a los macrólidos, cuyo mecanismo de acción es la inhibición de la síntesis proteica por medio de la subunidad 505 ribosomal de las bacterias y se ha descrito que presenta un efecto inmunomodulador.

Los estudios RECOVERY demostraron que el uso de azitromicina, en dosis estándar de 500 mg diarios por vía oral, durante 10 días por vía intravenosa, hasta la remisión de enfermedad en pacientes hospitalizados por COVID-19 o en combinación con cloroquina/hidroxicloroquina, no mejoró la supervivencia ni profilaxis de la enfermedad por el virus SARS-CoV-2; por lo que la OMS descartó su uso como blanco terapéutico de la COVID-19. ${ }^{38}$

La importancia del uso adecuado de antibióticos es indispensable para el manejo de sobreinfecciones bacterianas en neumonía severa por COVID-19, de tal modo que se evite la resistencia a los distintos antibióticos. ${ }^{39}$

\section{Ivermectina}

Es un antiparasitario potente activo contra ectoparásitos y nemátodos intestinales, tisulares y algunas filarias.

Los informes de los estudios in vitro contra el virus SARSCoV-2 sugieren que la ivermectina actúa inhibiendo las proteínas de transporte nuclear del hospedero (importina alfa/beta-1), que forman parte del transporte intracelular que los virus secuestran para potenciar la infección, suprimiendo la respuesta antiviral del hospedero; además de interferir con la adhesión de la proteína $\mathrm{S}$ del virión con la membrana celular del hospedero (Figura 3). Sin embargo, las dosis para alcanzar este efecto en cultivos son mayores a las dosis máximas aceptadas para el ser humano, por lo que no existe evidencia a la fecha del uso a favor de este medicamento como fármaco terapéutico contra la COVID-19. ${ }^{34}$

\section{INMUNOMODULADORES}

\section{Dexametasona}

Los glucocorticoides han sido utilizados en síndromes relacionados con COVID-19. Tales como SARS, síndrome respiratorio del Medio Oriente, influenza severa y neumonía adquirida en la comunidad. Sin embargo, la evidencia de este grupo farmacológico es débil para estas enfermedades por la falta de estudios controlados aleatorizados. ${ }^{40}$

En el Reino Unido se realizó el estudio RECOVERY aleatorizado, en donde se estudiaron 6,425 sujetos divididos en dos grupos. En el primero se evaluó el uso del manejo convencional de pacientes hospitalizados y en el segundo, el manejo convencional en combinación con 6 mg de dexametasona al día por un periodo de siete días. ${ }^{34}$ El mayor beneficio del uso de dexametasona para disminuir la mortalidad al día 28 se observó en aquellos pacientes que recibieron soporte ventilatorio y también en aquellos que fueron reclutados una semana después del inicio de la enfermedad, lo que sugiere que el estadio de la enfermedad pudo haber sido dominado por elementos inmunopatológicos, con una replicación viral activa en un segundo plano.

No se encontraron beneficios en pacientes que no requerían oxígeno suplementario, por lo que no se recomienda su uso en estas personas. ${ }^{41}$ 


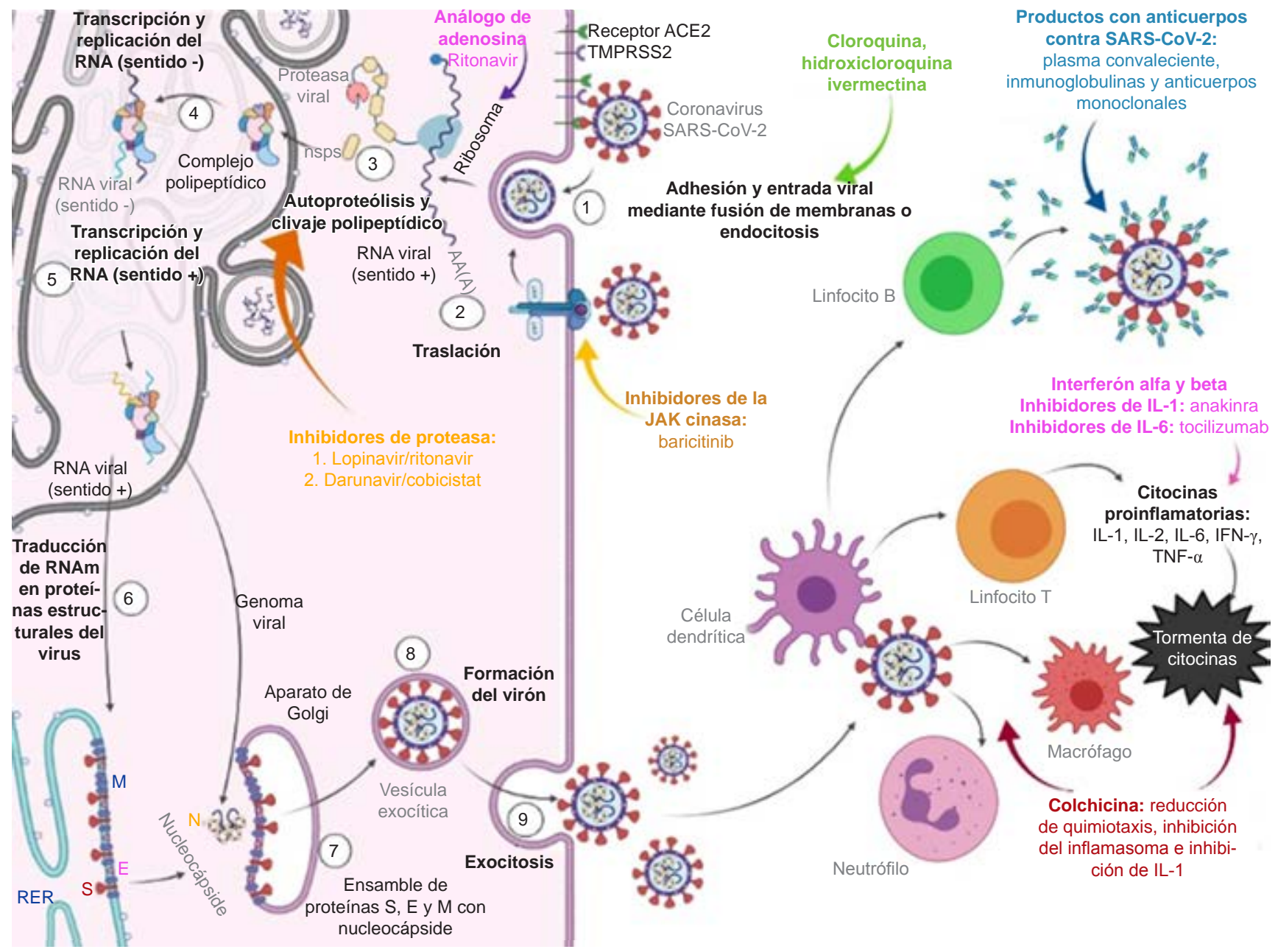

Figura 3: Blancos terapéuticos de los distintos fármacos implicados en el tratamiento de la infección por el virus SARS-CoV-2 durante su proceso patogénico.

ACE2 = enzima convertidora de angiotensina-2; TMPRSS2 = proteasa transmembrana de serina-2; RNA = ácido ribonucleico; $\mathrm{M}=$ proteína membranal; $\mathrm{S}$ = proteína espiga; $\mathrm{E}$ = envoltura; $\mathrm{N}$ = nucleocápside; $\mathrm{RER}$ = retículo endoplásmico rugoso; IL = interleucina; IFN = interferón, $\mathrm{TNF}=$ factor de necrosis tumoral.

Se recomienda el uso de dexametasona en pacientes con enfermedad severa o grave que requieran oxígeno suplementario o soporte ventilatorio a una dosis de $6 \mathrm{mg}$ por 10 días o hasta el alta hospitalaria.

También es importante la monitorización de eventos adversos, tales como hiperglucemia o el aumento del riesgo de infecciones bacterianas y fúngicas, glaucoma, cataratas, retención de líquidos, hipertensión, aumento de peso, osteoporosis, entre otras.

\section{Inhibidores de la IL-6}

La interleucina 6 es liberada en respuesta a infecciones y estimula las vías proinflamatorias como parte de una respuesta inflamatoria aguda. ${ }^{34}$
El tocilizumab y sarilumab son anticuerpos monoclonales que inhiben a los receptores de unión a la membrana plasmática celular y al receptor soluble de la IL-6. El uso clínico de estos fármacos para el tratamiento de la COVID-19 ha sido descrito en distintos ensayos controlados aleatorizados, con resultados principalmente negativos..$^{27,42}$ No obstante, los investigadores de la Plataforma Adaptativa, Multifactorial e Integrada para Estudios Aleatorizados de Neumonía Adquirida en la Comunidad (REMAP-CAP, por sus siglas en inglés) realizaron otro estudio clínico aleatorizado en el que obtuvieron resultados que contrastan con los previos; en este protocolo participaron 803 pacientes críticos con COVID-19 divididos en tres grupos. El primero fue compuesto por 353 personas que recibieron tocilizumab, 8 mg por kilogramo de peso (dosis máxima de 800 
$\mathrm{mg}$ ), con una dosis de repetición 12 a 24 horas después según el estado clínico del paciente; el segundo grupo fue compuesto por 48 personas, quienes recibieron 400 mg de sarilumab en una única ocasión; en el tercer grupo participaron 402 personas que conformaron el grupo control. Cabe mencionar que 93\% de los participantes de este estudio recibieron dexametasona como tratamiento coadyuvante, debido a que los resultados preliminares del estudio RECOVERY ya habían sido publicados. ${ }^{43}$

Se encontró que en pacientes críticos con COVID-19 el uso de antagonistas del receptor de interleucina 6 fue efectivo al aumentar los días libres de soporte orgánico, tanto ventilatorio como circulatorio en comparación al tratamiento convencional con glucocorticoides sin otro inmunomodulador. ${ }^{43}$

Otro estudio aleatorizado en el que participaron 249 pacientes para el grupo que recibió tocilizumab y 128 pacientes en el grupo placebo, se observó una baja probabilidad de progresión a ventilación mecánica en la cohorte que recibió tocilizumab; sin embargo, no se demostró mejoría en la supervivencia. ${ }^{44}$

El Centro de Control de Enfermedades (CDC, por sus siglas en inglés) recomienda el uso de tocilizumab en combinación con dexametasona en pacientes hospitalizados que presentan una rápida descompensación respiratoria, tales como pacientes que se encuentren dentro de sus primeras 24 horas en una $\mathrm{UCl}$ y que requieren ventilación mecánica o ventilación de alto flujo; o en pacientes no admitidos en una $\mathrm{UCl}$ en los cuales sus requerimientos de oxígeno incrementan rápidamente. ${ }^{26}$

\section{Colchicina}

La colchicina es un medicamento antiinflamatorio que se usa para tratar una variedad de afecciones, ${ }^{45,46}$ cuando se administra al comienzo del curso de COVID-19, estos mecanismos pueden mitigar o prevenir potencialmente las manifestaciones de la enfermedad asociadas con la inflamación, el ensayo de paciente ambulatorios COLCORONA mostró una ligera reducción en las hospitalizaciones en el subconjunto de pacientes ambulatorios, cuyo diagnóstico se confirmó mediante PCR para SARS-CoV-2, no se demostró eficacia en el tratamiento para pacientes hospitalizados. Los principales efectos adversos observados de la colchicina son: diarrea, náuseas, vómitos, calambres, dolor abdominal, hinchazón y pérdida del apetito. ${ }^{47}$

\section{Fluvoxamina}

Es un inhibidor selectivo de la recaptación de serotonina que se une al receptor sigma-1 en las células inmunes, lo que resulta en una producción reducida de citocinas inflamatorias. ${ }^{32,46}$
No hay datos suficientes para la recomendación a favor o en contra del uso de fluvoxamina como tratamiento de COVID-19, los estudios realizados hasta el momento no muestran resultados significativos. ${ }^{34,49,50}$

Interferones (Alfa, Beta)

Los interferones son una familia de citocinas con propiedades antivirales. No se recomienda el uso de interferones para el tratamiento en pacientes con COVID-19 grave o severo, ${ }^{51}$ existe muy poca información para recomendar el uso de interferón beta leve y moderado $(<7$ días desde iniciados los síntomas). ${ }^{52}$ Los estudios no han demostrado ningún beneficio de los interferones en pacientes con otras infecciones por coronavirus (MERS y SARS) que tienen una enfermedad grave o crítica. Además, los interferones tienen toxicidades importantes que superan el potencial de beneficio. ${ }^{53}$

\section{Inhibidores de IL-1}

No hay datos suficientes para la recomendación a favor o en contra del uso de inhibidores de la interleucina (IL)-1.54 Sin embargo, en el metaanálisis de Barkas se demostró que la Anakinra es una terapia eficaz y segura para disminuir la necesidad de ventilación mecánica invasiva, así como para reducir la mortalidad de los pacientes hospitalizados por COVID-19 severo con un riesgo mínimo de infecciones nosocomiales agregadas en las unidades de terapia intensiva. ${ }^{55}$

\section{Inhibidores de la JAK cinasa}

Esta opción terapéutica es considerada porque puede prevenir la fosforilación de proteínas clave, como son las citocinas proinflamatorias (IL-6), ${ }^{56}$ los inhibidores de la cinasa de Janus (JAK) y los que interfieren con la fosforilación de las proteínas transductoras de señales y activadoras de la transcripción (STAT), ${ }^{57,58}$ involucradas en la transducción de señales que conduce a la activación inmune y, por ende, a la inflamación.

La inmunosupresión inducida por esta clase de fármacos podría reducir potencialmente la inflamación y las inmunopatologías asociadas observadas en pacientes con COVID-19. Además, los inhibidores de JAK, en particular el baricitinib, tienen una actividad antiviral directa teórica a través de la interferencia con la endocitosis viral, lo que puede prevenir la entrada y la infección de las células susceptibles. ${ }^{59}$

Sin embargo, no hay datos suficientes que recomienden a favor o en contra el uso de estos fármacos para el tratamiento de COVID-19 en pacientes hospitalizados, cuando se pueden usar corticosteroides, cuando existe la rara circunstancia de que no se pueden usar corticoste- 
roides se recomienda usar baricitinib en combinación con remdesivir, pero no se recomienda el uso de estos fármacos individualmente. ${ }^{60}$

\section{ANTITROMBÓTICOS}

Existe una gran variedad de antitrombóticos, los cuales se dividen en: anticoagulantes orales, heparinas, inhibidores del factor Xa (indirectos- Fondaparinux), inhibidores directos de la trombina, antiplaquetarios y trombolíticos.

Las heparinas son el grupo de antitrombóticos mencionados en múltiples estudios que han demostrado una mejor eficacia en el tratamiento para pacientes infectados por SARS-CoV-2. ${ }^{61,62}$

\section{Heparinas}

Heparina no fraccionada (HNF): ésta, junto con las heparinas de bajo peso molecular (HBPM), son las de elección si se requiere un efecto anticoagulante rápido. Su uso es intrahospitalario, siendo su mecanismo de acción la unión a la antitrombina mediante un pentasacárido, que acelera más de mil veces su capacidad para inactivar los factores Ila y Xa. Esta heparina tiene una disponibilidad muy variable, vida media corta y efectos variables en cada paciente. Su monitoreo es mediante la medición de TTPa con rangos de 1.5 a 2.5 veces el del control, así el riesgo de sangrado está directamente relacionado con la dosis administrada. ${ }^{63}$

Heparina de bajo peso molecular (HBPM): entre ellas se encuentra la enoxaparina. Son un grupo heterogéneo de sustancias obtenidas de la HNF y cuyo efecto depende, igualmente, de su unión a la antitrombina, teniendo más capacidad de inhibir al factor Xa. Como ventajas muestra una mayor biodisponibilidad, una vida media más larga, un efecto antitrombótico más eficaz y mayor seguridad con menos efectos secundarios. Se administra dos veces al día y no requiere de exámenes de laboratorio para su control. ${ }^{64}$

Es importante mencionar que dependiendo si el paciente se encuentra hospitalizado y es considerado un caso moderado, se pueden administrar dosis profilácticas de enoxaparina 40 mg cada 24 horas vía subcutánea (SC) en pacientes sin factores de riesgo para trombosis, $1 \mathrm{mg} / \mathrm{kg}$ cada 24 horas vía $\mathrm{SC}$ en pacientes con factores de riesgo de trombosis; mientras que en casos graves se administra dosis terapéutica 1 $\mathrm{mg} / \mathrm{kg}$ cada 12 horas vía SC en fase aguda y $1.5 \mathrm{mg} / \mathrm{kg}$ cada 24 horas vía SC para dosis de mantenimiento. ${ }^{64}$

\section{TRATAMIENTOS CON ANTICUERPOS CONTRA SARS-CoV-2}

La mayor parte de pacientes con COVID-19 producen anticuerpos neutralizantes contra el SARS-CoV-2 aproxima- damente 10 días posterior a la aparición de la enfermedad, y se observan niveles de anticuerpos más elevados en aquellos pacientes que desarrollan enfermedad grave. ${ }^{34}$

Por lo que se han realizado estudios e investigaciones de posibles terapias contra el virus SARS-CoV-2 a través de productos que generen anticuerpos específicos contra el virus (Figura 3). La actividad neutralizante del plasma de los pacientes con COVID-19 se correlacionó con la magnitud de las respuestas de los anticuerpos a las proteínas $\mathrm{S}$ y $\mathrm{N}$ del virión del SARS-CoV-2, sin embargo, no demostraron disminución en la mortalidad. Los anticuerpos monoclonales dirigidos a la proteína $S$ tienen el potencial de prevenir la infección por SARS-CoV-2, así como aliviar los síntomas y limitar la progresión de la enfermedad grave en pacientes con COVID-19 de leve a moderada, especialmente en aquellos que aún no han desarrollado una respuesta endógena de anticuerpos. ${ }^{34}$

\section{Plasma de pacientes convalecientes}

Se refiere a productos plasmáticos de pacientes recuperados de COVID-19 que contienen anticuerpos contra el virus SARS-CoV-2 para ser administrados en pacientes hospitalizados para provocar la supresión del virus y modificar la respuesta inflamatoria. ${ }^{34}$

Este tipo de tratamiento no ha mostrado resultados a favor de su uso como terapia contra la COVID-19; por lo tanto, los ensayos RECOVERY finalizaron en enero del 2021, debido a que no mostraron evidencia significativa en la disminución de la mortalidad a los 28 días tras su empleo y con una eficacia de $18 \%$ similar a la atención médica común.

Un estudio realizado en Argentina con 160 pacientes adultos mayores demostró que la administración de títulos altos de anticuerpos neutralizantes de pacientes recuperados de la enfermedad a pacientes adultos mayores con enfermedad leve/moderada redujo la progresión de la enfermedad por COVID-19; ${ }^{65}$ sin embargo, su uso aún se encuentra limitado para fines de investigación.

\section{Anticuerpos monoclonales}

Como su nombre lo indica, son anticuerpos monoclonales neutralizantes que se unen a diferentes epítopos del dominio del receptor de la proteína S del SARS-CoV-2. Su empleo ha sido aprobado por la FDA como tratamiento de emergencia en pacientes con COVID-19 leve o moderado, no hospitalizados y sin uso de oxígeno, tanto pacientes adultos y pediátricos, con edad mínima de 12 años y al menos $40 \mathrm{~kg}$ de peso corporal. ${ }^{34,66}$ La combinación de estos anticuerpos monoclonales ha mostrado reducir la progresión de la enfermedad a estadios graves que requie- 
ren uso de oxígeno suplementario y/u hospitalización. Se han utilizado varias combinaciones, entre las que destacan:

1. Bamlanivimab más etesevimab $(700 / 1,400 \mathrm{mg})^{66}$

2. Casirivimab más imdevimab $(1,200 / 1,200$ mg)

Entre los efectos adversos más frecuentes destacan náuseas, diarrea, cefalea, prurito, vómito, rash, fiebre e hipersensibilidad al medicamento. ${ }^{34}$

En caso de recibir este tipo de terapia, la vacunación contra el virus SARS-CoV-2 deberá ser diferida a 90 días posterior a uso de anticuerpos monoclonales. ${ }^{34}$

\section{Inmunoglobulinas}

Consisten en preparaciones concentradas de anticuerpos derivados del plasma obtenido de distintos individuos que se han recuperado de la COVID-19, que podría suprimir la actividad del virus y modificar la respuesta inflamatoria. ${ }^{34} \mathrm{El}$ uso de inmunoglobulinas específicas para otras infecciones virales ha demostrado ser segura y eficaz; sin embargo, actualmente no hay datos clínicos sobre el uso de éstas para la COVID-19. Los riesgos potenciales en la aplicación de estas preparaciones pueden incluir reacciones transfusionales (reacciones febriles, hipotensivas, hemolíticas, infecciosas, entre otras) o la potenciación de la infección dependiente de los anticuerpos. ${ }^{34}$

\section{SUPLEMENTOS VITAMÍNICOS}

\section{Vitamina D}

Previenen las infecciones respiratorias en personas de diferentes edades, ${ }^{67-69}$ particularmente en aquellas con deficiencias previas de vitamina $\mathrm{D}$, por medio de la producción de defensinas y catelicidinas, así como incrementar las concentraciones de citocinas antiinflamatorias. ${ }^{70} \mathrm{Sin}$ embargo, los estudios realizados hasta el momento con vitamina D y pacientes infectados por SARS-CoV-2, no muestran mejoría en el pronóstico o la prevención contra este mismo. ${ }^{71}$

\section{Vitamina $\mathrm{C}$}

Previene el resfriado común, principalmente en aquellas personas bajo estrés físico extremo, reduciendo la duración y aliviando los síntomas. ${ }^{72-74}$ Ha demostrado la disminución del estado de gravedad en pacientes infectados con SARS-CoV-2, reflejado en la disminución de la duración de la sintomatología, ${ }^{75}$ así como una disminución en la mortalidad en pacientes en estado séptico. ${ }^{76}$

Dosis: $200 \mathrm{mg}-2,000 \mathrm{mg} / \mathrm{d} .^{76}$
Zinc

Disminuye el estado de gravedad de los pacientes con la reducción en los días de recuperación. ${ }^{75}$ Se propuso que inhibe la replicación de varios virus, entre ellos influenza y el SARS-CoV- $2,{ }^{78}$ y se ha asociado a la reducción de la mortalidad en tratamiento conjunto con hidroxicloroquina. ${ }^{79}$ Dosis: 6.8-9.5 mg. ${ }^{76}$

\section{CONCLUSIONES}

El conocimiento de las distintas dianas y terapias farmacológicas para el manejo de pacientes con COVID-19 resulta de vital importancia para el personal médico. El uso y desuso de algunos fármacos es un proceso en constante cambio a lo largo del control de la pandemia por el virus SARS-CoV-2 en las diferentes regiones del mundo. Sin embargo, la OMS ha aceptado varios tratamientos, siendo la dexametasona el estándar del tratamiento en pacientes con enfermedad moderada/severa. La OMS, la FDA y otras organizaciones han aprobado otros medicamentos para la evitar la progresión de la enfermedad y reducir la mortalidad, como es el uso combinado de remdesivir/baricitinib, anticuerpos monoclonales como bamlanivimab/etesevimab, oxígeno suplementario, la adopción de la posición prona del paciente y el uso de heparinas según la presencia de comorbilidades asociadas. Se debe tener especial cuidado y vigilancia en los efectos adversos de cada medicamento, valorando la respuesta a cada uno de ellos para la adecuada recuperación y alta del paciente del medio hospitalario.

Conflicto de intereses: Declaramos que ninguno de los autores presenta conflictos de intereses.

\section{REFERENCIAS}

1. V'kovski P, Kratzel A, Steiner S, Stalder H, Thiel V. Coronavirus biology and replication: implications for SARS-CoV-2. Nat Rev Microbiol. 2021; 19 (3): 155-170. doi: 10.1038/s41579-020-00468-6.

2. Hamming I, Timens W, Bulthuis ML, Lely AT, Navis G, van Goor H. Tissue distribution of ACE2 protein, the functional receptor for SARS coronavirus. A first step in understanding SARS pathogenesis. J Pathol. 2004; 203 (2): 631-637. doi: 10.1002/path.1570.

3. Rabi FA, Al Zoubi MS, Kasasbeh GA, Salameh DM, Al-Nasser AD. SARS-CoV-2 and coronavirus disease 2019: what we know so far. Pathogens. 2020; 9 (3): 231. doi: 10.3390/pathogens9030231.

4. Bhaskar S, Sinha A, Banach M, Mittoo S, Weissert R, Kass JS et al. Cytokine storm in COVID-19-immunopathological mechanisms, clinical considerations, and therapeutic approaches: the REPROGRAM consortium position paper. Front Immunol. 2020; 11: 1648. doi: 10.3389/fimmu.2020.01648.

5. McIntosh K. Coronavirus disease 2019 (COVID-19): epidemiology, virology, clinical features, diagnosis, and prevention. In: Hirsch MS, ed. UpToDate. Waltham, MA: UpToDate; 2020 [Modificado 23 de abril de 2021; consultado 25 de abril de 2021]. Available in: https:// www.uptodate.com 
6. Gandhi RT, Lynch JB, Del Rio C. Mild or moderate Covid-19. N Engl J Med. 2020; 383 (18): 1757-1766. doi: 10.1056/NEJMcp2009249.

7. Marini JJ, Gattinoni L. Management of COVID-19 respiratory distress. JAMA. 2020; 323 (22): 2329-2330. doi: 10.1001/jama.2020.6825.

8. Thompson BT, Chambers RC, Liu KD. Acute respiratory distress syndrome. N Engl J Med. 2017; 377 (6): 562-572. doi: 10.1056/ NEJMra1608077.

9. Barrot L, Asfar P, Mauny F, Winiszewski H, Montini F, Badie J et al. Liberal or conservative oxygen therapy for acute respiratory distress syndrome. N Engl J Med. 2020; 382 (11): 999-1008. doi: 10.1056/ NEJMoa1916431.

10. Montejo JC, García de Lorenzo A, Marco P, Ortiz C. Manual de medicina intensiva. 5a ed. Barcelona: Elsevier; 2017.

11. OPS. Algoritmo de manejo de pacientes con sospecha de infección por COVID-19 en el primer nivel de atención y en zonas remotas de la Región de las Américas. Organización Panamericana de la Salud, 2020. [Acceso 28 de abril de 2021] Disponible en: https://iris.paho.org/ bitstream/handle/10665.2/52501/OPSIMSEIHCOVID-19200012 spa.pdf? sequence $=6$ \&isAllowed $=y$

12. Masclans JR, Pérez-Terán P, Roca O. The role of high flow oxygen therapy in acute respiratory failure. Med Intensiva. 2015; 39 (8): 505-515. doi: 10.1016/j.medin.2015.05.009.

13. Ni YN, Luo J, Yu H, Liu D, Liang BM, Liang ZA. The effect of high-flow nasal cannula in reducing the mortality and the rate of endotracheal intubation when used before mechanical ventilation compared with conventional oxygen therapy and noninvasive positive pressure ventilation. A systematic review and meta-analysis. Am J Emerg Med. 2018; 36 (2): 226-233. doi: 10.1016/j.ajem.2017.07.083.

14. Frat JP, Thille AW, Mercat A, Girault C, Ragot S, Perbet S et al. Highflow oxygen through nasal cannula in acute hypoxemic respiratory failure. N Engl J Med. 2015; 372 (23): 2185-2196. doi: 10.1056/ NEJMoa1503326.

15. Kapil S, Wilson JG. Mechanical ventilation in hypoxemic respiratory failure. Emerg Med Clin North Am. 2019; 37 (3): 431-444. doi: 10.1016/j.emc.2019.04.005.

16. Fan E, Del Sorbo L, Goligher EC, Hodgson CL, Munshi L, Walkey AJ et al. An Official American Thoracic Society/European Society of Intensive Care Medicine/Society of Critical Care Medicine Clinical Practice Guideline: mechanical ventilation in adult patients with acute respiratory distress syndrome. Am J Respir Crit Care Med. 2017; 195 (9): 1253-1263. doi: 10.1164/rccm.201703-0548ST.

17. Sartini C, Tresoldi M, Scarpellini P, Tettamanti A, Carco F, Landoni G et al. Respiratory parameters in patients with COVID-19 after using noninvasive ventilation in the prone position outside the Intensive Care Unit. JAMA. 2020; 323 (22): 2338-2340. doi: 10.1001/ jama.2020.7861.

18. Guérin C, Reignier J, Richard JC, Beuret P, Gacouin A, Boulain T et al. Prone positioning in severe acute respiratory distress syndrome. N Engl J Med. 2013; 368 (23): 2159-2168. doi: 10.1056/NEJMoa1214103.

19. World Health Organization. Clinical management clinical management: living guidance COVID-19. World Health Organization; 2021.

20. Bednarczyk JM, Fridfinnson JA, Kumar A, Blanchard L, Rabbani R, Bell $D$ et al. Incorporating dynamic assessment of fluid responsiveness into goal-directed therapy: a systematic review and metaanalysis. Crit Care Med. 2017; 45 (9): 1538-1545. doi: 10.1097/ CCM.0000000000002554.

21. Brown RM, Wang L, Coston TD, Krishnan NI, Casey JD, Wanderer JP et al. Balanced crystalloids versus saline in sepsis. A secondary analysis of the SMART clinical trial. Am J Respir Crit Care Med. 2019; 200 (12): 1487-1495. doi: 10.1164/rccm.201903-0557OC.

22. Han H, Yang L, Liu R, Liu F, Wu KL, Li J et al. Prominent changes in blood coagulation of patients with SARS-CoV-2 infection. Clin Chem Lab Med. 2020; 58 (7): 1116-1120. doi: 10.1515/cclm-2020-0188.
23. Tang N, Bai H, Chen X, Gong J, Li D, Sun Z. Anticoagulant treatment is associated with decreased mortality in severe coronavirus disease 2019 patients with coagulopathy. J Thromb Haemost. 2020; 18 (5): 1094-1099. doi: 10.1111/jth.14817.

24. Beigel JH, Tomashek KM, Dodd LE, Mehta AK, Zingman BS, Kalil AC et al. Remdesivir for the treatment of Covid-19 - final report. N Engl J Med. 2020; 383 (19): 1813-1826. doi: 10.1056/NEJMoa2007764.

25. Goldman JD, Lye DCB, Hui DS, Marks KM, Bruno R, Montejano R et al. Remdesivir for 5 or 10 days in patients with severe Covid-19. N Engl J Med. 2020; 383 (19): 1827-1837. doi: 10.1056/NEJMoa201530

26. Interleukin-6 Inhibitors. COVID-19 treatment guidelines. 2021. Available in: https://www.covid19treatmentguidelines.nih.gov/ immunomodulators/interleukin-6-inhibitors/

27. Stone JH, Frigault MJ, Serling-Boyd NJ, Fernandes AD, Harvey L, Foulkes AS et al. Efficacy of tocilizumab in patients hospitalized with Covid-19. N Engl J Med. 2020; 383 (24): 2333-2344. doi: 10.1056/ NEJMoa2028836.

28. WHO Rapid Evidence Appraisal for COVID-19 Therapies (REACT) Working Group, Sterne JAC, Murthy S, Diaz JV, Slutsky AS, Villar J et al. Association between administration of systemic corticosteroids and mortality among critically ill patients with COVID-19: a meta-analysis. JAMA. 2020; 324 (13): 1330-1341. doi: 10.1001/jama.2020.17023.

29. Tomazini BM, Maia IS, Cavalcanti AB, Berwanger O, Rosa RG, Veiga $V C$ et al. Effect of dexamethasone on days alive and ventilator-free in patients with moderate or severe acute respiratory distress syndrome and COVID-19: the CoDEX randomized clinical trial. JAMA. 2020; 324 (13): 1307-1316. doi: 10.1001/jama.2020.17021.

30. Grein J, Ohmagari N, Shin D, Diaz G, Asperges E, Castagna A et al. Compassionate use of remdesivir for patients with severe Covid-19. N Engl J Med. 2020; 382 (24): 2327-2336. doi: 10.1056/ NEJMoa2007016.

31. Beigel JH, Tomashek KM, Dodd LE, Mehta AK, Zingman BS, Kalil AC et al. Remdesivir for the treatment of Covid-19 - final report. N Engl J Med. 2020; 383 (19): 1813-1826. doi: 10.1056/NEJMoa2007764.

32. Goldman JD, Lye DCB, Hui DS, Marks KM, Bruno R, Montejano R et al. Remdesivir for 5 or 10 days in patients with severe Covid-19. N Engl J Med. 2020; 383 (19): 1827-1837. doi: 10.1056/NEJMoa2015301.

33. Kalil AC, Patterson TF, Mehta AK, Tomashek KM, Wolfe CR, Ghazaryan $V$ et al. Baricitinib plus remdesivir for hospitalized adults with Covid-19. N Engl J Med. 2021; 384 (9): 795-807. doi: 10.1056/ NEJMoa2031994.

34. National Institutes of Health. Coronavirus Disease 2019 (COVID-19) treatment guidelines [Internet]. Bethesda (MD): National Institutes of Health (US); 2021. [Consult Apr 27 2021] Available in: www. covid19treatmentguidelines.nih.gov

35. RECOVERY Collaborative Group. Lopinavir-ritonavir in patients admitted to hospital with COVID-19 (RECOVERY): a randomised, controlled, open-label, platform trial. Lancet. 2020; 396 (10259): 1345-1352. doi: 10.1016/S0140-6736(20)32013-4.

36. Mitja O, Corbacho-Monné M, Ubals M, Alemany A, Suñer C, Tebé $C$ et al. A cluster-randomized trial of hydroxychloroquine for prevention of Covid-19. N Engl J Med. 2021; 384 (5): 417-427. doi: 10.1056/ NEJMoa2021801.

37. Cavalcanti AB, Zampieri FG, Rosa RG, Azevedo LCP, Veiga VC, Avezum A et al. Hydroxychloroquine with or without azithromycin in mild-to-moderate Covid-19. N Engl J Med. 2020; 383 (21): 20412052. doi: 10.1056/NEJMoa2019014.

38. RECOVERY Collaborative Group. Azithromycin in patients admitted to hospital with COVID-19 (RECOVERY): a randomised, controlled, open-label, platform trial. Lancet. 2021; 397 (10274): 605-612. doi: 10.1016/S0140-6736(21)00149-5.

39. PRINCIPLE Trial Collaborative Group. Azithromycin for community treatment of suspected COVID-19 in people at increased risk of an adverse clinical course in the UK (PRINCIPLE): a randomised, 
controlled, open-label, adaptive platform trial. Lancet. 2021; 397 (10279): 1063-1074. doi: 10.1016/S0140-6736(21)00461-X.

40. Thompson BT, Chambers RC, Liu KD. Acute respiratory distress syndrome. N Engl J Med. 2017; 377 (6): 562-572. doi: 10.1056/ NEJMra1608077.

41. RECOVERY Collaborative Group, Horby P, Lim WS, Emberson JR, Mafham M, Bell JL et al. Dexamethasone in hospitalized patients with Covid-19. N Engl J Med. 2021; 384 (8): 693-704. doi: 10.1056/ NEJMoa2021436.

42. Rosas IO, Brau N, Waters M, Go RC, Hunter BD, Bhagani S et al. Tocilizumab in hospitalized patients with severe Covid-19 pneumonia. N Engl J Med. 2021; 384 (16): 1503-1516. doi: 10.1056/ NEJMoa2028700.

43. REMAP-CAP Investigators, Gordon AC, Mouncey PR, Al-Beidh F, Rowan KM, Nichol AD et al. Interleukin-6 receptor antagonists in critically ill patients with Covid-19. N Engl J Med. 2021; 384 (16): 1491-1502. doi: 10.1056/NEJMoa2100433.

44. Salama C, Han J, Yau L, Reiss WG, Kramer B, Neidhart JD et al. Tocilizumab in patients hospitalized with Covid-19 pneumonia. $N$ Engl J Med. 2021; 384 (1): 20-30. doi: 10.1056/NEJMoa2030340.

45. van Echteld I, Wechalekar MD, Schlesinger N, Buchbinder R, Aletaha D. Colchicine for acute gout. Cochrane Database Syst Rev. 2014; (8): CD006190. doi: 10.1002/14651858.CD006190.pub2.

46. Tardif JC, Bouabdallaoui N, L'Allier PL, Gaudet D, Shah B, Pillinger $\mathrm{MH}$ et al. Efficacy of colchicine in non-hospitalized patients with COVID-19. The preprint server for health service. medRxiv. 2021. Available in: https://doi.org/10.1101/2021.01.26.21250494

47. Colchicina (Colcrys) [prospecto]. Administración de Alimentos y Medicamentos. 2012. Disponible en: https://www.accessdata.fda. gov/drugsatfda docs/label/2014/022352s017lbl.pdf

48. Rosen DA, Seki SM, Fernández-Castañeda A, Beiter RM, Eccles JD, Woodfolk JA et al. Modulation of the sigma-1 receptor-IRE1 pathway is beneficial in preclinical models of inflammation and sepsis. Sci Transl Med. 2019; 11 (478): eaau5266. doi: 10.1126/scitranslmed.aau5266.

49. Seftel D, Boulware DR. Prospective cohort of fluvoxamine for early treatment of coronavirus disease 19. Open Forum Infect Dis. 2021; 8 (2): ofab050. doi: 10.1093/ofid/ofab050.

50. Lenze EJ, Mattar C, Zorumski CF, Stevens A, Schweiger J, Nicol GE et al. Fluvoxamine vs placebo and clinical deterioration in outpatients with symptomatic COVID-19: a randomized clinical trial. JAMA. 2020; 324 (22): 2292-2300. doi: 10.1001/jama.2020.22760.

51. Zhou Q, MacArthur MR, He X, Wei X, Zarin P, Hanna BS et al. Interferon- $\alpha 2 b$ Treatment for COVID-19 is associated with improvements in lung abnormalities. Viruses. 2020; 13 (1): 44. doi: 10.3390/v13010044.

52. Davoudi-Monfared E, Rahmani H, Khalili H, Hajiabdolbaghi $M$, Salehi M, Abbasian L et al. A randomized clinical trial of the efficacy and safety of interferon $\beta-1 \mathrm{a}$ in treatment of severe COVID-19. Antimicrob Agents Chemother. 2020; 64 (9): e01061-20. doi: 10.1128/AAC.01061-20.

53. COVID-19 Treatment Guidelines. Interferons. 2020. Available in: https://www.covid19treatmentguidelines.nih.gov/ immunomodulators/interferons/

54. Huet T, Beaussier H, Voisin O, Jouveshomme S, Dauriat G, Lazareth I et al. Anakinra for severe forms of COVID-19: a cohort study. Lancet Rheumatol. 2020; 2 (7): e393-e400. doi: 10.1016/S26659913(20)30164-8.

55. Barkas F, Ntekouan SF, Kosmidou M, Liberopoulos E, Liontos A, Milionis $\mathrm{H}$. Anakinra in hospitalized non-intubated patients with coronavirus disease 2019: a systematic review and meta-analysis. Rheumatology (Oxford). 2021: keab447. doi: 10.1093/rheumatology/ keab447.

56. Zhang W, Zhao Y, Zhang F, Wang Q, Li T, Liu Z et al. The use of anti-inflammatory drugs in the treatment of people with severe coronavirus disease 2019 (COVID-19): The Perspectives of clinical immunologists from China. Clin Immunol. 2020; 214: 108393. doi: 10.1016/j.clim.2020.108393.

57. Babon JJ, Lucet IS, Murphy JM, Nicola NA, Varghese LN. The molecular regulation of Janus kinase (JAK) activation. Biochem J. 2014; 462 (1): 1-13. doi: 10.1042/BJ20140712.

58. Bousoik E, Montazeri Aliabadi H. "Do we know jack" about Jak? A closer look at JAK/STAT signaling pathway. Front Oncol. 2018; 8: 287. doi: 10.3389/fonc.2018.00287.

59. Smith AD, Kim Yl, Refsum H. Is folic acid good for everyone? Am J Clin Nutr. 2008; 87 (3): 517-533. doi: 10.1093/ajen/87.3.517.

60. COVID-19 Treatment Guidelines. Kinase inhibitors. 2021. Available in: https://www.covid19treatmentguidelines.nih.gov/ immunomodulators/kinase-inhibitors/

61. Moores LK, Tritschler T, Brosnahan S, Carrier M, Collen JF, Doerschug $\mathrm{K}$ et al. Prevention, diagnosis, and treatment of VTE in patients with coronavirus disease 2019: CHEST Guideline and Expert Panel Report. Chest. 2020; 158 (3): 1143-1163. doi: 10.1016/j.chest.2020.05.559.

62. Llitjos JF, Leclerc M, Chochois C, Monsallier JM, Ramakers M, Auvray $\mathrm{M}$ et al. High incidence of venous thromboembolic events in anticoagulated severe COVID-19 patients. J Thromb Haemost. 2020; 18 (7): 1743-1746. doi: 10.1111/jth.14869.

63. Mammen, EF. Current development in antithrombotic therapy. Semin Thromb Hemost. 2004; 30 (6): 605-607. Available in: https://doi. org/10.1055/s-2004-861501

64. SETH. Recomendaciones de tromboprofilaxis y tratamiento antitrombótico en pacientes con COVID-19. Sociedad Española de Trombosis y Hemostasia. 2020. Disponible en: https://www.covid-19. seth.es/recomendaciones-de-tromboprofilaxis-y-tratamientoantitrombotico-en-pacientes-con-covid-19/

65. Libster R, Pérez Marc G, Wappner D, Coviello S, Bianchi A, Braem $\checkmark$ et al. Early high-titer plasma therapy to prevent severe Covid-19 in older adults. N Engl J Med. 2021; 384 (7): 610-618. doi: 10.1056/ NEJMoa2033700.

66. Lilly Investors. Lilly's bamlanivimab and etesevimab together reduced hospitalizations and death in phase 3 trial for early COVID-19. 2021. Available in: https://investor.lilly.com/news-releases/news-releasedetails/lillys-bamlanivimab-and-etesevimab-together-reduced

67. Martineau AR, Jolliffe DA, Greenberg L, Aloia JF, Bergman P, Dubnov-Raz G et al. S102 Vitamin d supplementation to prevent acute respiratory infections: systematic review and meta-analysis of individual participant data. Thorax. 2016; 71 (Suppl 3): A60.2-A61. Available in: doi.org/10.1136/thoraxjnl-2016-209333.108

68. Charan J, Goyal JP, Saxena D, Yadav P. Vitamin D for prevention of respiratory tract infections: A systematic review and meta-analysis. J Pharmacol Pharmacother. 2012; 3 (4): 300-303. doi: 10.4103/0976500X.103685

69. Xiao L, Xing C, Yang Z, Xu S, Wang M, Du H et al. Vitamin D supplementation for the prevention of childhood acute respiratory infections: a systematic review of randomised controlled trials. $\mathrm{Br} J$ Nutr. 2015; 114 (7): 1026-1034. doi: 10.1017/S000711451500207X

70. Bonvecchio A, Pacheco S, Irizarry L, Herrera M, Tijerina M, Bernal J et al. Recomendaciones de micronutrientes para grupos vulnerables en contexto de desnutrición, durante la pandemia de COVID-19 en Latinoamérica. Arch Latinoam Nutr. 2019; 69: 259-273.

71. Murai IH, Fernandes AL, Sales LP, Pinto AJ, Goessler KF, Duran CSC et al. Effect of a single high dose of vitamin D3 on hospital length of stay in patients with moderate to severe COVID-19: a randomized clinical trial. JAMA. 2021; 325 (11): 1053-1060. doi: 10.1001/ jama.2020.26848.

72. Ran L, Zhao W, Wang H, Zhao Y, Bu H. Vitamin C as a supplementary therapy in relieving symptoms of the common cold: a meta-analysis of 10 randomized controlled trials. Biomed Res Int. 2020; 2020: 8573742. doi: 10.1155/2020/8573742. 
73. Hemila $\mathrm{H}$, Chalker E. Vitamin $\mathrm{C}$ for preventing and treating the common cold. Cochrane Database Syst Rev. 2013; 2013 (1): CD000980. doi: 10.1002/14651858.CD000980.pub4.

74. Carr AC, Maggini S. Vitamin C and immune function. Nutrients. 2017; 9 (11): 1211. doi: 10.3390/nu9111211.

75. Thomas S, Patel D, Bittel B, Wolski K, Wang Q, Kumar A et al. Effect of high-dose zinc and ascorbic acid supplementation vs usual care on symptom length and reduction among ambulatory patients with SARSCoV-2 infection: the COVID a to Z randomized clinical trial. JAMA Netw Open. 2021; 4 (2): e210369. doi: 10.1001/jamanetworkopen.2021.0369.

76. Comité Internacional para la Elaboración de Consensos y Estandarización en Nutriología (CIENUT). Posición de expertos sobre el manejo nutricional del coronavirus COVID-19. Lima: Fondo editorial IIDENUT. 2020. Disponible en: https://cienut.org/ comite_internacional/declaraciones/pdf/declaracion2.pdf
77. Fowler AA, Truwit JD, Hite RD, Morris PE, DeWilde C, Priday A et al. Effect of vitamin C infusion on organ failure and biomarkers of inflammation and vascular injury in patients with sepsis and severe acute respiratory failure: the CITRIS-ALI randomized clinical trial. JAMA. 2019; 322 (13): 1261-1270. doi: 10.1001/ jama.2019.11825.

78. te Velthuis AJ, van den Worm SH, Sims AC, Baric RS, Snijder EJ, van Hemert MJ. $\mathrm{Zn}(2+)$ inhibits coronavirus and arterivirus RNA polymerase activity in vitro and zinc ionophores block the replication of these viruses in cell culture. PLoS Pathog. 2010; 6 (11): e1001176. doi: 10.1371/journal.ppat.1001176.

79. Frontera JA, Rahimian JO, Yaghi S, Liu M, Lewis A, de Havenon A et al. Treatment with zinc is associated with reduced in-hospital mortality among COVID-19 patients: a multi-center cohort study. Res Sq. 2020: rs.3.rs-94509. doi: 10.21203/rs.3.rs-94509/v1. 